\title{
Riesgo por tsunami en la ciudad de San Antonio: un aporte a gobiernos locales
}

\author{
Fabiola Barrenechea R.* \\ María Fernanda Ferrada** \\ Moisés Jara***
}

Recibido el 3 de agosto de 2015; aceptado el 7 agosto de 2016

\begin{abstract}
The geological location of Chile in the circle of the South Pacific, where the coastline runs parallel to the subduction zone between the plates Nazca and South American, has caused the tsunamis are recurring, still a major problem for the population, due to its complexity and sudden occurrence. It is for this reason that the determination of tsunami risk areas for the first response effect becomes a priority and urgent need to help the authorities to be timelier, efficient and effective decisions.

The study presented below is based on the development of a user-friendly methodology that enables municipalities with populations in the coastal edge; identify areas at risk of tsunami through a Geographic Information System. The methodology was applied in the municipality of San Antonio, Valparaíso Region. For development of cadastre information materiality of homes he raised, a digital elevation model and vector coverage concerning the commune socioeconomic information was used.
\end{abstract}

Key words: Risk, Vulnerability, Hazard, Tsunami risk, SIG.

* Escuela de Ingeniería en Geomensura y Cartografía, Universidad Bernardo O’Higgins, Avenida Viel 1497, Ruta 5 Sur, Santiago, Chile, correo electrónico: fabarren@gmail.com

** Escuela de Ingeniería en Geomensura y Cartografía, Universidad Bernardo O’Higgins, correo electrónico: maria_ferrada@hotmail.com

*** Escuela de Ingeniería en Geomensura y Cartografía, Universidad Bernardo O’Higgins, correo electrónico: moi.jara.lopez@gmail.com 


\section{Resumo}

A localização geológica do Chile, na zona de subducção entre as placas de Nazca e da América do Sul, tem feito com que os tsunamis sejam recorrentes, gerando grandes problemas para a população.

Este trabalho se baseia na proposta de uma metodologia de fácil aplicação, que permite que os municípios costeiros do país, identifiquem as áreas de risco a tsunamis através de um Sistema de Informação Geográfica para gerir a primeira resposta a uma emergência. A metodologia foi aplicada no município de San Antonio, Região de Valparaíso. Seu desenvolvimento contemplou informação de cadastros materiais de moradias, modelo de elevação digital e coberturas vetoriais de informação socioeconômica do município.

Palavras-chave: risco, vulnerabilidade, risco de tsunami, SIG.

\section{Resumen}

La ubicación geológica de Chile, en la zona de subducción entre las Placas de Nazca y Sudamericana, ha hecho que los tsunamis sean recurrentes, causando una gran problemática para la población.

El presente trabajo se basa en la propuesta de una metodología de fácil aplicación, que permita a los municipios costeros del país, identificar zonas de riesgo de tsunamis por medio de un Sistema de Información Geográfica, para gestionar la primera respuesta frente a una situación de emergencia. La metodología se aplicó en la Comuna de San Antonio, Región de Valparaíso. Su desarrollo contempló información de catastros de materialidad de las viviendas, modelo digital de elevación y coberturas vectoriales de información socioeconómica referente a la comuna.

Palabras clave: riesgo, vulnerabilidad, riesgo de tsunami, SIG.

\section{Introducción}

A lo largo de la historia, numerosos fenómenos naturales han azotado el territorio chileno, impactando a su población y generando pérdidas económicas considerables que han hecho retroceder su desarrollo económico.

$\mathrm{Su}$ ubicación geológica emplazada en la zona de subducción de las Placas de Nazca y Sudamericana, ha traído como consecuencia la ocurrencia de numerosos eventos naturales tales como erupciones volcánicas y sismos de gran magnitud, los que han generado grandes impactos a la población. De acuerdo al Centro Sismológico Nacional, desde el año 1570 a la fecha, se han generado 113 terremotos con magnitudes superiores a 7.0 en la Escala de Richter, lo que da cuenta de la gran actividad sísmica que tiene esta zona. Por otra parte, la configuración paralela de una línea de costa (con más de 4,000 km de longitud), con la zona de subducción, 
ha favorecido la ocurrencia de al menos 70 tsunamis que han destruido infraestructura y poblados costeros en más de una ocasión.

Según Lockridge (1985), citado por el Servicio Hidrográfico y Oceanográfico de la Armada (SHOA), un tsunami se define como "una serie de ondas oceánicas generadas por un disturbio impulsivo en el océano, o en un pequeño y conectado cuerpo de agua". Este disturbio puede ser generado por desplazamientos abruptos del fondo oceánico, causados por terremotos, deslizamientos de tierra submarinos o de la línea de la costa, erupciones volcánicas y explosiones.

\section{Concepto de Riesgo}

De acuerdo con la definición de la Organización de las Naciones Unidas (ONU), el riesgo es la probabilidad de consecuencias perjudiciales o pérdidas esperadas, resultado de interacciones entre amenazas de origen natural o antropogénicas y condiciones de vulnerabilidad (Oficina de las Naciones Unidas para la Reducción de Riesgos Naturales - UNIDSR, 2010). Se tiene entonces que el riesgo es la probabilidad de que una amenaza se convierta en desastres, dependiendo de la vulnerabilidad de un territorio:

\section{RIESGO $=$ AMENAZA $x$ VULNERABILIDAD}

Sin presencia humana (personas, bienes o servicios) no se genera una situación de riesgo puesto que ningún elemento está bajo amenaza. Solamente con la presencia humana, en su sentido amplio, puede considerarse una situación de riesgo. Por ello, el riesgo de impacto potencial de un evento debe ser valorado a partir de la interrelación existente entre los aspectos físicos y los aspectos sociales. En términos simples, se tiene entonces que riesgo es la probabilidad de que una amenaza pueda generar un impacto negativo en la población que se encuentra vulnerable. Consecuentemente, el riesgo es la probabilidad de ocurrencia de un fenómeno y de los daños asociados (Chardon y González, 2002).

Dado que la amenaza es constante (la ocurrencia de los fenómenos naturales no desaparece, ni se pueden aminorar), la única forma de trabajar en la reducción del riesgo de desastres es a través de la vulnerabilidad.

La vulnerabilidad está íntimamente ligada a los procesos sociales que se desarrollan en las áreas propensas y usualmente tiene que ver con la fragilidad, la susceptibilidad o la falta de resiliencia de la población ante amenazas de diferente índole. Podría decirse entonces que los desastres son eventos socio-ambientales cuya materialización es el resultado de la construcción social del riesgo (Cardona et al., 2013).

Para efectos de medición de la vulnerabilidad, esta posee subdivisiones en donde se destaca la del autor Wilches-Chaux (1984), definiendo diferentes tipos de 
vulnerabilidad. Entre las más importantes se encuentra la vulnerabilidad física que hace referencia a la localización de la población en zona de riesgo físico, condición provocada, generalmente, por la pobreza y la falta de oportunidades para una ubicación de menor riesgo (condiciones ambientales y de los ecosistemas, localización de asentamientos humanos en zonas de riesgo); la vulnerabilidad económica en la cual se observa una relación indirecta entre los ingresos en los niveles nacional, regional, local o poblacional y el impacto de los fenómenos físicos extremos. Es decir, la pobreza aumenta el riesgo de desastre (desempleo, insuficiencia de ingresos, explotación, inestabilidad laboral, dificultad de acceso a los servicios de educación, salud, ocio); y por último, la vulnerabilidad social donde se produce un grado deficiente de organización y cohesión interna de la sociedad bajo riesgo que limita su capacidad de prevenir, mitigar o responder a situaciones de desastres (tipo de acceso al saneamiento ambiental, nutrición infantil, servicios básicos, que permitan la recuperación de los daños ocurridos).

Para efecto de este estudio y su posterior medición y metodología nos enfocaremos en los términos de vulnerabilidad física y social utilizados por WilchesChaux (1984), los cuales los definiremos como:

- Vulnerabilidad Física: hace referencia a la localización o ubicación de la población, en una determinada zona de riesgo. También se determina por la ubicación de las construcciones y la precariedad que puedan tener para soportar algunos desastres (materialidad, estado, entre otras).

- Vulnerabilidad Social: se entenderá como las condiciones de las personas dentro de sus hogares, ya sea cantidad de integrantes, rango etario (niños, adultos, tercera edad).

\section{Concepto de tsunami}

Un maremoto, conocido principalmente por su extranjerismo como Tsunami - -del japonés tsu (puerto o bahía) y nami (ola)_, "consiste en una serie de olas de gran energía, tamaño y velocidad que se irradian hacia el exterior desde un foco" (SHOA, 2012). Según la Oficina Nacional de Emergencia del Ministerio de Interior y Seguridad Pública (ONEMI, 2014), un tsunami se define como "un fenómeno físico que se origina por sismos que ocurren bajo o cerca del fondo oceánico, remociones en masa, derrumbes y erupciones volcánicas submarinas, y se manifiesta en la costa como una gran masa de agua que puede causar inundación y destrucción en las zonas costeras".

De acuerdo al SHOA (2012), la mayoría de los tsunamis que han afectado las costas de Chile han sido generados por sismos de magnitud superior a 7.0 Ricther, cuyo epicentro se ha localizado en el fondo marino o cercano a éste. Para que un sismo origine un tsunami, el fondo marino debe ser deformado abruptamente en 
sentido vertical por un desplazamiento tectónico, de modo que la masa de agua es impulsada fuera de su equilibrio normal. Cuando ésta trata de recuperar su equilibrio, se generan las olas. Así, el tamaño del tsunami estará determinado por la magnitud de la deformación vertical del fondo marino.

El impacto y la magnitud de los efectos de un tsunami en áreas costeras, según Inamura y Lida, citados por Monge (1993) va a depender de una serie de factores físicos y de la existencia o no de emplazamientos humanos. En la Tabla 1 se describen escalas de grados de tsunamis, su poder destructor, sus efectos en la costa y daños ocasionados.

Tabla 1

Escala de grado de tsunami

\begin{tabular}{cccl}
\hline $\begin{array}{c}\text { Grado } \\
\text { mag }=m\end{array}$ & $\begin{array}{c}\text { Altura de ola }(H) \text { en } \\
\text { metros }\end{array}$ & $\begin{array}{c}\text { Cota máxima de } \\
\text { inundación }(R) \text { en } \\
\text { metros }\end{array}$ & \multicolumn{1}{c}{ Descripción de los daños } \\
\hline 0 & $01-02$ & $1-1.5$ & No produce daños \\
1 & $02-05$ & $2-3$ & $\begin{array}{l}\text { Casas inundadas y botes } \\
\text { destruidos son arrastrados }\end{array}$ \\
2 & $05-10$ & $4-6$ & $\begin{array}{l}\text { Personas, barcos y casas son barridos } \\
3\end{array}$ \\
$10-30$ & $8-12$ & $\begin{array}{l}\text { Daños extendidos a lo largo de } 400 \\
\text { kilómetros de la costa }\end{array}$ \\
4 & & $16-24$ & $\begin{array}{l}\text { Daños extendidos sobre más de } 500 \\
\text { kilómetros a lo largo de la línea } \\
\text { costera }\end{array}$ \\
\hline
\end{tabular}

Fuente: Inamura y Lida, transcrita por Wiegel, 1970.

Dentro de los eventos de mayor impacto se puede mencionar el terremoto del 22 de mayo de 1960, cuyo epicentro se ubicó en las cercanías de Valdivia, el más fuerte que se tenga registro en la historia del mundo, con una magnitud de 9.5 Richter. Este terremoto tsunamigénico, tuvo un poder destructivo de tal intensidad que hundió la costa de Valdivia 4 metros bajo el nivel del mar y el tsunami producido se propagó por todo el Océano Pacífico afectando, entre otras zonas del sur de Chile, Hawai y Japón, dejando 2,000 muertos en Chile y más de 3,000 en las costas del Pacífico, sin contar la gran cantidad de viviendas destruidas (Strahler, 1994).

El catálogo italiano de tsunamis del Instituto Nacional de Geofísica y Vulcanología de Italia (Istituto Nazionale Geofisica e Vulcanologia, INGV) muestra los tsunamis más destructivos en la última década. 
Tabla 2

Tsunamis de mayor poder destructivo en la última década

\begin{tabular}{clll}
\hline & \multicolumn{1}{c}{ Fecha } & \multicolumn{1}{c}{ Origen } & \multicolumn{1}{c}{ Causa } \\
\hline 1 & 26 de diciembre de 2004 & Sudeste Asiático & Sismo de 9.3 Richter \\
2 & 17 de julio de 2006 & Indonesia & Sismo de 7.7 Richter \\
3 & 02 de abril de 2007 & Islas Salomón & Sismo de 8.0 Richter \\
4 & 29 de septiembre de 2009 & Samoa & Sismo de 7.7 Richter \\
5 & 27 de febrero de 2010 & Chile & Sismo de 8.8 Richter \\
6 & 11 de marzo de 2011 & Japón & Sismo de 8.9 Richter \\
\hline
\end{tabular}

Fuente: Istituto Nazionale Geofisica e Vulcanologia.

\section{Metodología}

\section{Área de estudio}

La ciudad de San Antonio forma parte de la Comuna y Provincia del mismo nombre, que se inserta en la Región de Valparaíso, ubicada en la zona central de Chile. Se localiza a 108 kilómetros al Oeste de Santiago (Ilustre Municipalidad de San Antonio). Presenta un clima mediterráneo con influencia marítima, cuya temperatura media anual es de $13.5^{\circ} \mathrm{C}$ y precipitaciones con un total anual $325 \mathrm{~mm}$. Dentro de su geomorfología se pueden diferenciar tres grandes unidades: cerros, planicies y ribera del Río Maipo, los que se ordenan secuencialmente en una línea de norte a sur, formando una conurbación lineal contenida hacia el poniente por el sector portuario, y hacia el oriente por una meseta superior en la zona alta de la ciudad aún no consolidada; plan de borde, donde se aprecia la planicie costera que constituye el sector portuario y la Meseta superior que se extiende desde las quebradas del Estero Arévalo y Aguas Buenas por el norte, hasta el Estero El Sauce por el sur, es una zona extensa de pendientes suaves. Junto con lo anterior, se caracteriza por presentar planicies costeras con relieves escalonados, producto de la abrasión marina y procesos geotectónicos. Así, es posible distinguir tres niveles de terrazas, con alturas relativas del orden de los 200-250 metros sobre el nivel del mar (msnm), para la terraza alta; $100-150 \mathrm{msnm}$, para la terraza media y $0-50 \mathrm{msnm}$, para la terraza baja (Araya-Vergara, 1976).

De acuerdo con las cifras otorgadas por el Instituto Nacional de Estadísticas del año (INE, 2002), el total de la población de la Comuna de San Antonio es de 87,205 habitantes. Además se considera una cifra proyectada de 99,868 habitantes para el año 2012 , teniendo una variación de un $14.50 \%$, de acuerdo al último censo oficial en Chile. 


\section{Pasos metodológicos}

La identificación de zonas de riesgo de tsunami, se trabajó con procesamientos de información digital de San Antonio, a través de herramientas SIG, de acuerdo a las siguientes variables:

\section{Vulnerabilidad}

Se acotaron a dos tipos: vulnerabilidad física de la vivienda y la vulnerabilidad socioeconómica y demográfica.

Las variables más representativas para la medición del nivel de vulnerabilidad física y sociodemográfica se escogieron en función de las metodologías propuestas por Mardones y Vidal (2001), caso aplicado a inundación fluvial. De esta manera se determinaron las siguientes variables y sus ponderaciones. Cabe destacar que las ponderaciones de cada una de las vulnerabilidades, fueron establecidas por medio del juicio experto:

a) Vulnerabilidad Física:

i) Materialidad de la Vivienda: definida por el grado de resistencia a la inundación por tsunami. Los tipos de materialidad que se consideraron son: hormigón, hormigón armado (material pesado); ladrillo, piedra, bloques prefabricados (albañilería mixta); madera, internit o adobe (materiales ligeros).

ii) Estado de Conservación de la Vivienda: estado en que se encuentra una estructura. Se clasificaron en buena, regular y deteriorada.

iii) Cercanía a la Costa: se determinaron por estimación propia entre la distancia aproximada mínima desde la costa hasta una zona residencial, y los últimos sectores residenciales de San Antonio, ubicados bajo la cota menor a 30msnm. La última variable que se consideró dentro de la vulnerabilidad física de las viviendas es la cercanía a la costa. Esta variable se vuelve fundamental a la hora de determinar el riesgo por tsunami, ya que se relaciona directamente con la exposición que presentan las viviendas y su población. El valor se obtuvo por medio del cálculo del geocentroide de cada una de las manzanas por medio de SIG. No se realizó el cálculo para cada una de las viviendas dado que la diferencia de las distancias entre las viviendas de una misma manzana, en promedio, es residual para el cálculo de su vulnerabilidad.

Así, las variables anteriores se relacionan por medio de la siguiente fórmula:

$$
V F=\left(M V^{*} 6\right)+(E C * 4)+(C C * 8)
$$


Donde:

$\mathrm{VF}=$ Vulnerabilidad Física de la Vivienda

$\mathrm{MV}=$ Materialidad de la Vivienda

$\mathrm{EC}=$ Estado de Conservación

$\mathrm{CC}=$ Cercanía a la Costa

Tabla 3

Ponderación y Nivel de Vulnerabilidad Física

\begin{tabular}{lcccc}
\hline & $\begin{array}{c}\text { Materialidad } \\
\text { de la } \\
\text { vivienda }\end{array}$ & $\begin{array}{c}\text { Estado de } \\
\text { conservación }\end{array}$ & $\begin{array}{c}\text { Cercanía } \\
\text { a la costa }\end{array}$ & $\begin{array}{c}\text { Nivel de } \\
\text { vulnerabilidad }\end{array}$ \\
\hline Ponderación & 6 & 4 & 8 & Rango \\
Alto 3 & $\begin{array}{c}\text { Materiales } \\
\text { ligeros }\end{array}$ & Deteriorada & $<400$ metros & $44-56$ \\
Medio 2 & $\begin{array}{c}\text { Albañilería } \\
\text { mixta } \\
\text { Bajo 1 }\end{array}$ & Regular & $\begin{array}{c}401-800 \\
\text { metros }\end{array}$ & $31-43$ \\
& pesado & Buena & $>800$ metros & $18-30$ \\
\hline
\end{tabular}

Fuente: Elaboración de los autores.

b) Vulnerabilidad Socioeconómica y Demográfica:

i) Nivel Socio Económico: se consideraron las cinco clases sociales agrupadas en tres rangos establecidos: nivel socioeconómico $\mathrm{ABC} 1$ (menos vulnerable); $\mathrm{C} 2$ y C3 (similares estadísticamente, medianamente vulnerables); y los estratos D y E (considerados los más vulnerables).

ii) Cantidad de Habitantes por manzana: se utilizó la cantidad de viviendas multiplicado por 4, con el propósito de contar con un número más aproximado a la realidad, dado que el último censo válido consta con información del año 2002. El cálculo se utilizó en base al trabajo realizado por Carvajal (2010).

iii) Cantidad de Viviendas por Manzana: dato obtenido desde la Ilustre Municipalidad de San Antonio.

Definidos los ponderadores, la vulnerabilidad socioeconómica y demográfica se determinó por medio de la siguiente fórmula:

$$
V S D=(N S E * 8)+(C H * 10)+(C V * 6)
$$


Donde:

$\mathrm{VSD}=$ Vulnerabilidad Socioeconómica Demográfica

$\mathrm{NSE}=$ Nivel Socioeconómico

$\mathrm{CH}=$ Cantidad Habitantes

$\mathrm{CV}=\quad$ Cantidad Viviendas

Tabla 4

Ponderación y Nivel de Vulnerabilidad Socioeconómica y Demográfica

\begin{tabular}{lcccc}
\hline & $\begin{array}{c}\text { Nivel } \\
\text { Socioeconómico }^{1}\end{array}$ & $\begin{array}{c}\text { Cantidad de } \\
\text { habitantes }\end{array}$ & $\begin{array}{c}\text { Cantidad de } \\
\text { Viviendas }\end{array}$ & $\begin{array}{c}\text { Nivel de } \\
\text { Vulnerabilidad }\end{array}$ \\
\hline Ponderación & 8 & 10 & 6 & Rango \\
Alto 3 & D-E & $>400$ & $>80$ & $50-66$ \\
Medio 2 & C1-C2 & $201-400$ & $41-80$ & $33-49$ \\
Bajo 1 & ABC1 & $<200$ & $<40$ & $16-32$ \\
\hline
\end{tabular}

Fuente: Elaboración de los autores.

Calculada la Vulnerabilidad Física y la Vulnerabilidad Socioeconómica y Demográfica, se determinó la vulnerabilidad final de la siguiente manera:

$$
V=(V F * 4)+(V S D * 6)
$$

Donde:

$\mathrm{V}=\quad$ Vulnerabilidad

$\mathrm{VF}=\quad$ Vulnerabilidad Física

$\mathrm{VSD}=$ Vulnerabilidad Socioeconómica y Demográfica

\section{Amenaza}

Altitud

La altitud se consideró como un factor determinante para la amenaza, ya que va estrechamente relacionado con la inundación. Dado que no existen estudios específicos que indiquen la cota de inundación, la Armada de Chile a través del SHOA define la cota 30 como altitud máxima de inundación por tsunami. Es por esto que se consideró la cota de los $30 \mathrm{msnm}$, como cota máxima de inundación. Su ubicación se calculó por medio de ArcGis utilizando un Modelo Digital de Elevación

\footnotetext{
Los niveles socioecnómicos en Chile corresponden al poder adquisitivo de la población y se dividen en cinco clases: $\mathrm{ABC} 1$, definida como clase con alto poder adquisitivo, $\mathrm{C} 2$ y $\mathrm{C} 3$ definida como clase media y emergente, D y E como la clase de menos poder adquisitivo, por lo tanto la más vulnerable.
} 
DEM Aster GDEM, en formato Raster-GeoTiff, con una resolución espacial de 30 metros y resolución radiométrica de 16 bits, corregido y facilitado por el Centro de Investigación en Recursos Naturales (CIREN) del año 2009.

\section{Determinación del Riesgo}

Como ya se ha mencionado anteriormente, Riesgo se define como la relación de los factores de Amenaza y Vulnerabilidad. Por ello se desprende la siguiente fórmula:

$$
R=(V * 4)+(A * 6)
$$

Donde:

$\mathrm{R}=\quad$ Riesgo de tsunami

$\mathrm{V}=\quad$ Vulnerabilidad

$\mathrm{A}=\quad$ Amenaza de la vivienda

En donde la matriz multicriterio para la obtención de rangos es la siguiente:

Tabla 5

Nivel de Riesgo

\begin{tabular}{lc}
\hline Niveles de riesgo & Rangos \\
\hline Alta & $26-33$ \\
Media & $18-25$ \\
Baja & $10-17$ \\
\hline
\end{tabular}

Fuente: Elaboración de los autores.

\section{Resultados}

De acuerdo a la información levantada en terreno de cada una de las viviendas ubicadas en la zona de inundación, ${ }^{2}$ se logró determinar que las viviendas ubicadas en esta zona presentan en su mayoría materialidad ligera y albañilería mixta, predominando esta última con un $50 \%$ de las viviendas. Este tipo de construcción se distribuye de manera uniforme en toda la zona de estudio. Las viviendas de material ligero, por su parte, ocupan un $20 \%$ del total de viviendas, emplazándose princi-

2 Dado que el estudio contempla la identificación de zonas de riesgo de tsunami, se seleccionaron las manzanas que se ubican bajo la cota $30 \mathrm{msnm}$ dado que éste es un factor determinante en la definición de zonas de riesgo de por inundación, de acuerdo a lo establecido por el SHOA. De esta manera se obtuvieron 332 manzanas a catastrar y analizar. 


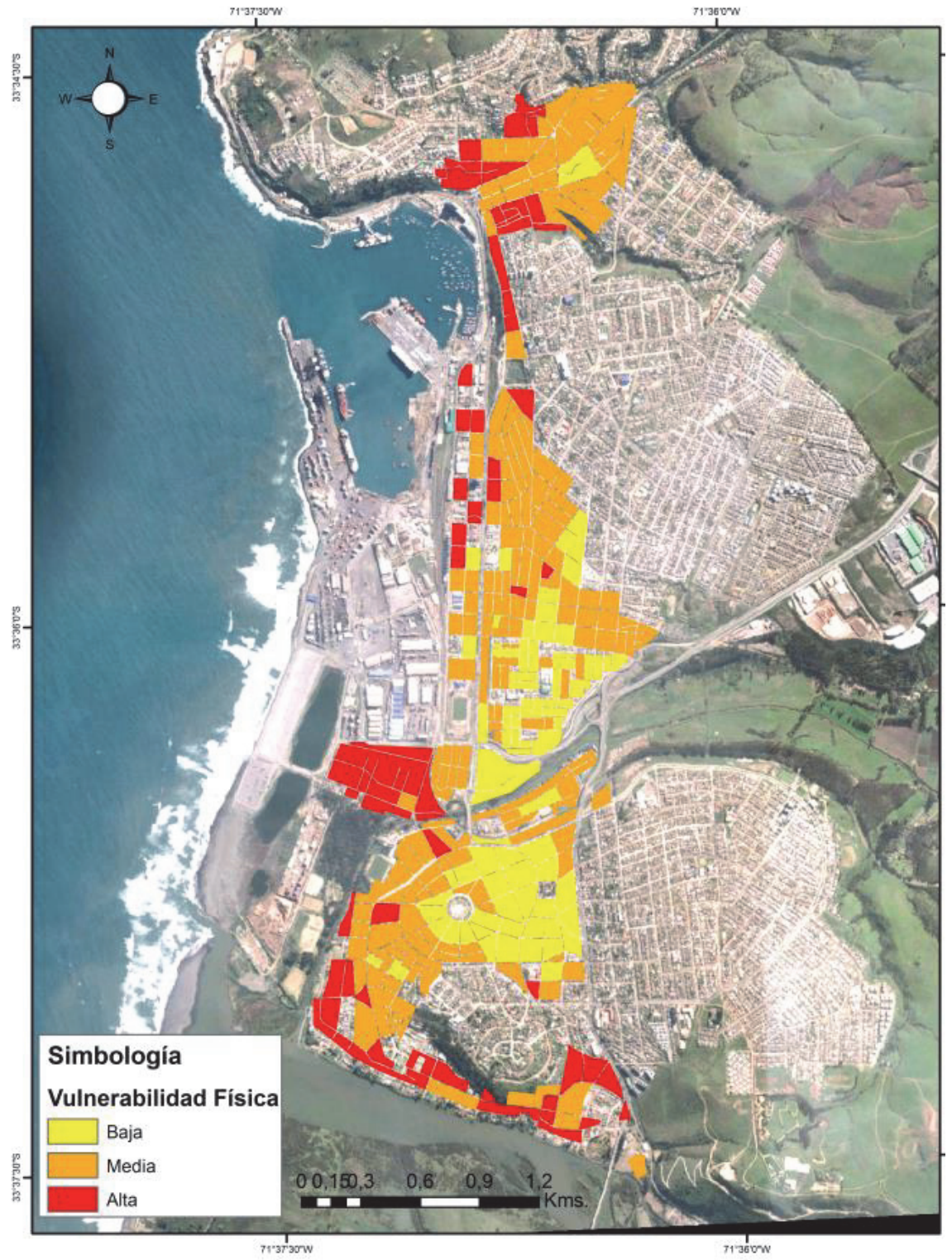

Figura 1. Mapa de Vulnerabilidad Física.

Fuente: elaboración del autor. 


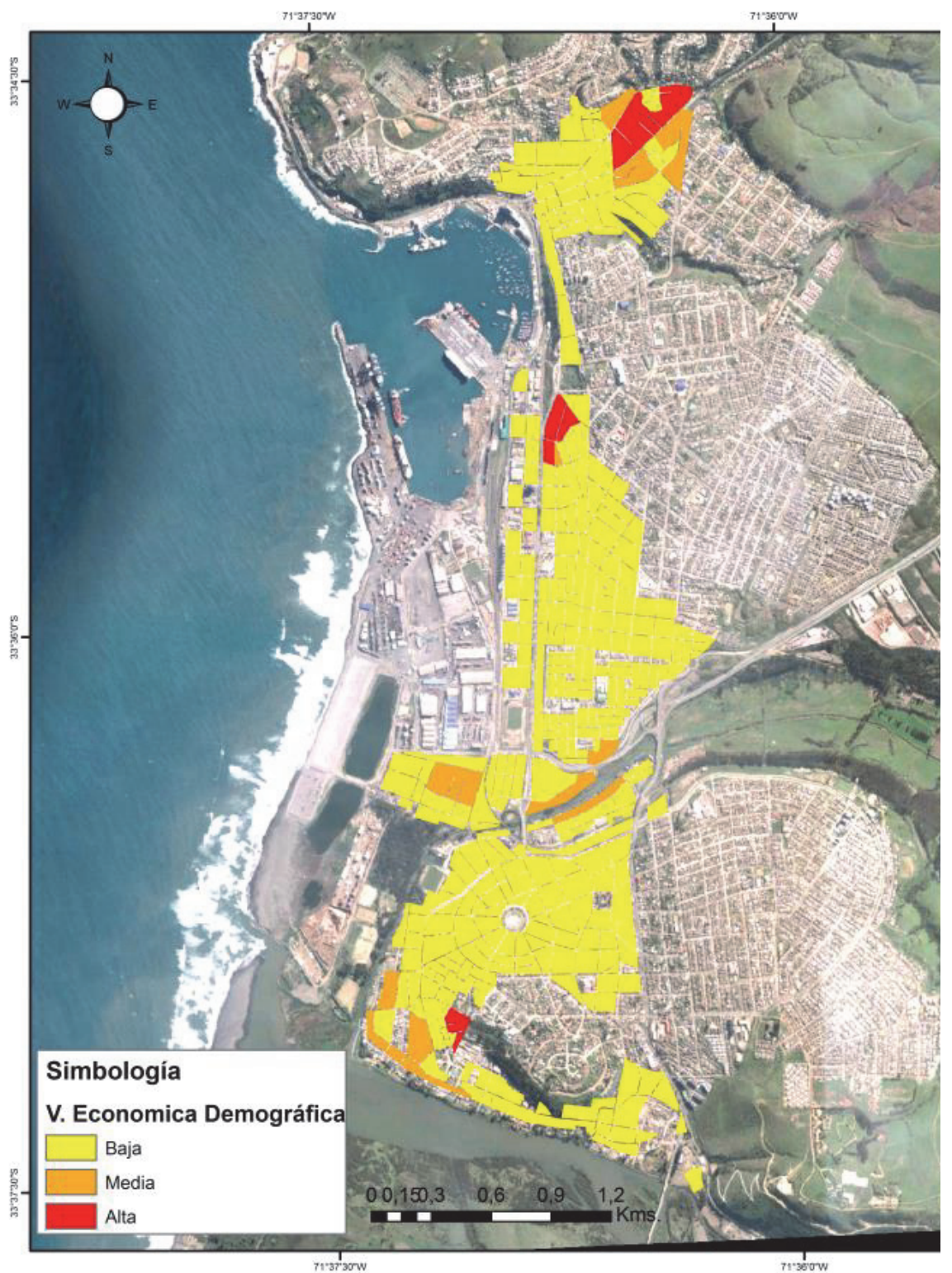

Figura 2. Mapa de Vulnerabilidad Económica-Demográfica.

Fuente: elaboración de los autores. 


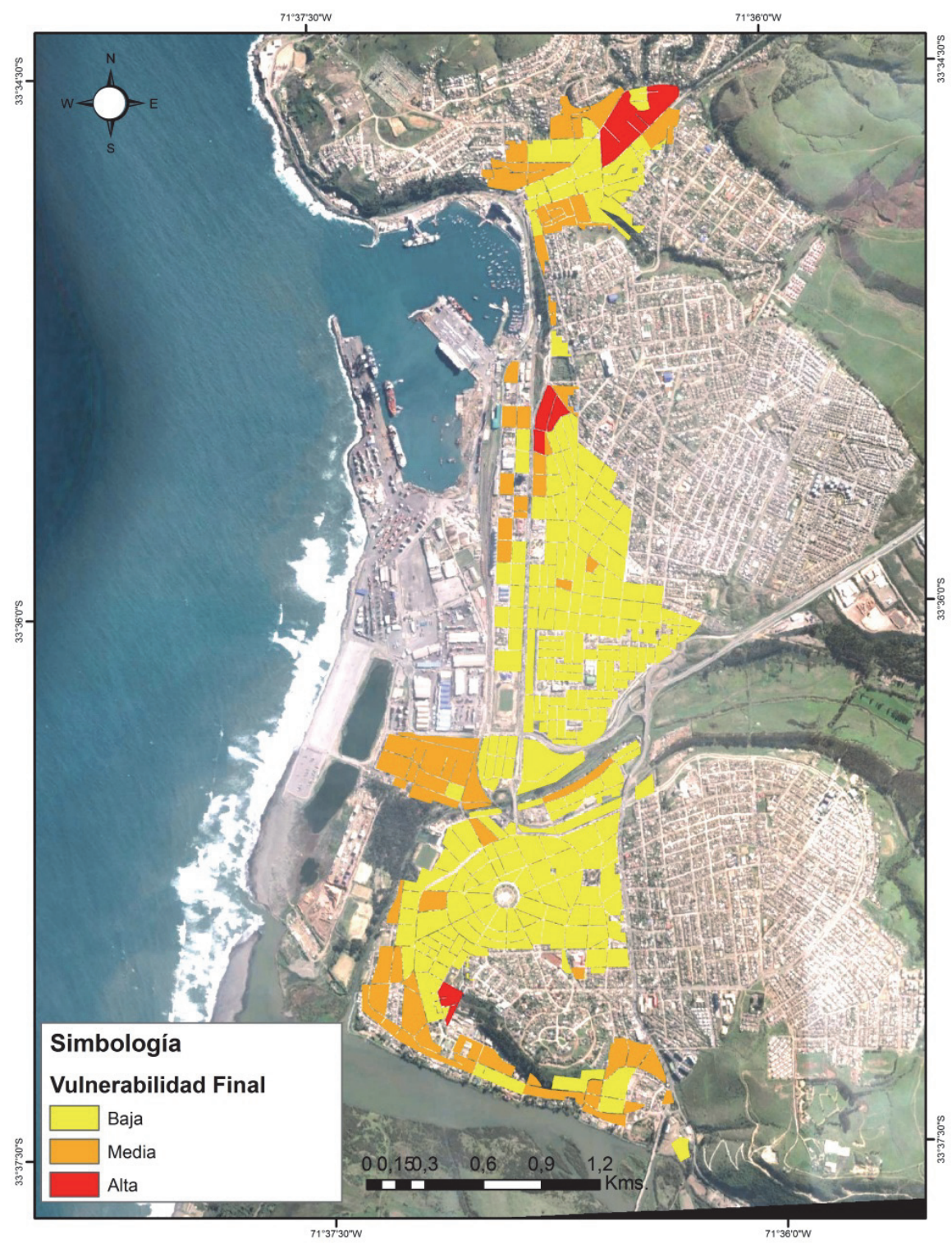

Figura 3. Mapa de Vulnerabilidad Final.

Fuente: elaboración del autor. 


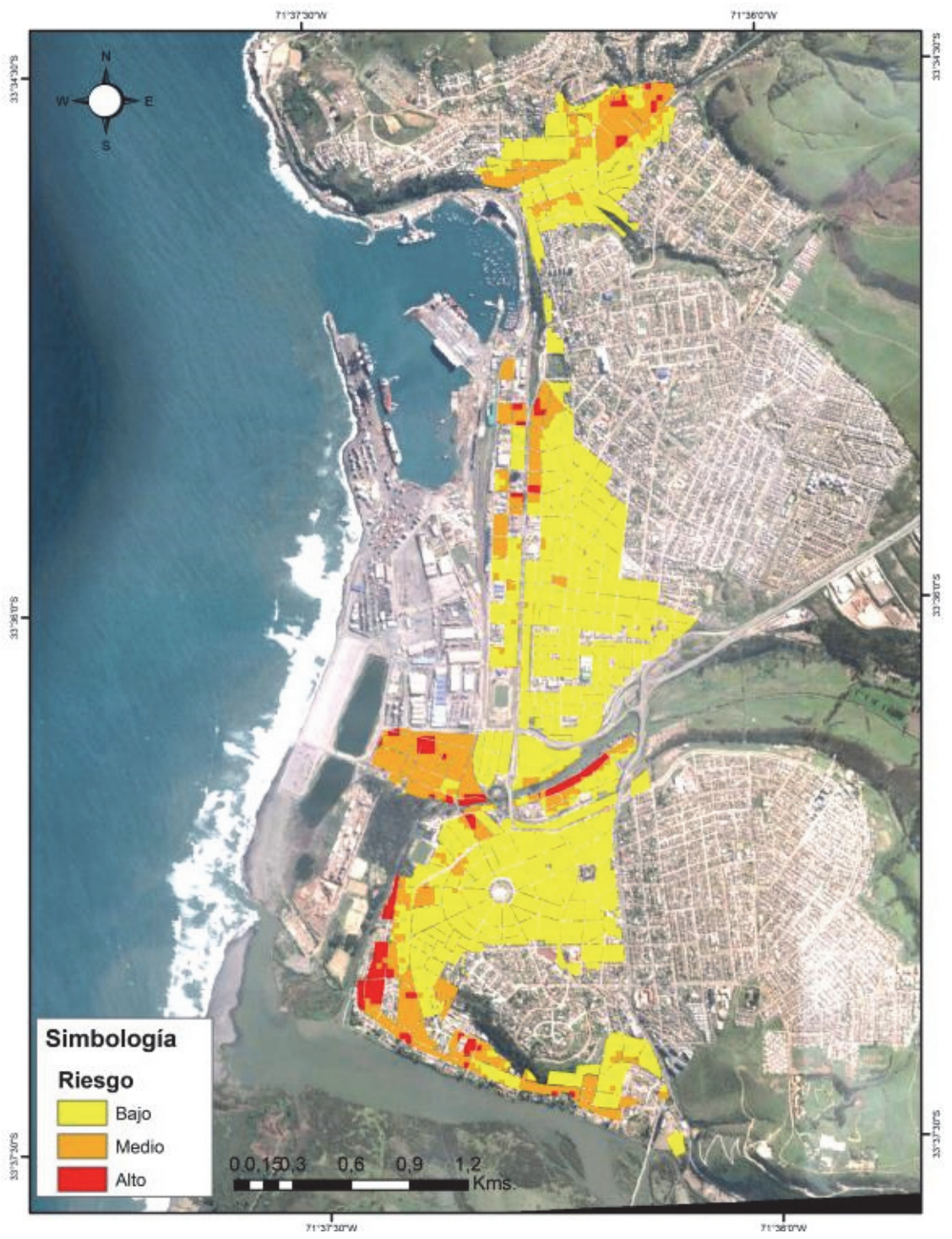

Figura 4. Mapa de Riesgo de Tsunami.

Fuente: elaboración de los autores. 
palmente hacia el norte del puerto. Las construcciones de material pesado son las que representan una menor cantidad, abarcando aproximadamente un 30\%. Lo anterior puede deberse a los factores socioeconómicos que presenta la ciudad. San Antonio se caracteriza por ser una comuna de bajos ingresos económicos, donde un gran porcentaje de población viaja a Santiago diariamente en busca de una fuente laboral más estable. La tasa de cesantía supera el 7\% siendo uno de los más altos a nivel nacional. Este hecho contribuye a que los estratos sociales y económicos que en la ciudad conviven, sean en su mayoría D y E, y en menor medida C3 a C2, existiendo un casi nulo porcentaje de población en la categoría $\mathrm{ABC} 1$. La escases de ingresos por parte de la población, ha ayudado a que la materialidad de las viviendas sean en su mayoría del tipo albañilería mixta o material ligero, donde su estado de conservación no siempre es el mejor. En relación a esto último, un $20 \%$ de las viviendas poseen un estado bueno, un 45\% regular y un 35\% de las viviendas se encuentra con un estado deteriorado.

De esta forma, considerando todas las variables anteriores, la vulnerabilidad física de las viviendas se presenta de acuerdo a la Figura 1. Se puede apreciar que sólo un 33\% de las manzanas estudiadas presenta una vulnerabilidad física alta y un $54 \%$ presenta una vulnerabilidad física media. Esto se debe principalmente a la variable de cercanía a la costa. La presencia del puerto en el borde costero de casi toda la ciudad, condiciona la variable evaluada de cercanía a la costa, donde su cálculo estableció niveles de vulnerabilidad bajo y medio para el $60 \%$ de las manzanas analizadas.

Para la estimación de la vulnerabilidad socioeconómica y demográfica, se trabajó con información sobre el nivel socioeconómico, cantidad de habitantes y la cantidad de viviendas por manzana. En el caso de la primera variable, se observa que cerca de un $50 \%$ de la población se encuentra categorizada con un nivel socioeconómico D y E, seguida por la clase $\mathrm{C} 2$ y C3. Esta categorización de la población se relaciona con el alto nivel de cesantía presente en la comuna, lo que influye directamente en la materialidad de las viviendas. Las familias de escasos recursos suelen construir sus viviendas en lugares de alto riesgo y de una materialidad poco adecuada a la alta exposición que presentan. De acuerdo al Informe Global de Evaluación sobre Reducción del Riesgo de Desastres de Naciones Unidas (2015), es común que en los países en vías de desarrollo, dado su acelerado crecimiento, se configuren nuevas condiciones de riesgo producto de factores como el crecimiento demográfico, migración, pobreza, desigualdad, una rápida urbanización y la ausencia de planificación en terrenos no aptos.

En el caso de la variable de vulnerabilidad socioeconómica y demográfica, la distribución de la concentración de población se muestra de manera uniforme, donde la población no supera las 200 personas por manzana. Destaca una mayor concentración en el sector norte de la ciudad y la ribera norte del Río Maipo, en el 
sector de la desembocadura. La baja concentración de población se condice con la baja cantidad de viviendas, las que en su mayoría no superan las 40 por manzana. A diferencia de las localidades aledañas, la condición de ciudad — puerto que presenta San Antonio-, ha generado que la ciudad no cuente con grandes playas que favorezcan el turismo, por lo que el fenómeno de vivienda de segunda residencia se da en menor grado que en el resto de las localidades del litoral central.

Estimadas las variables anteriores, la vulnerabilidad socioeconómica y demográfica se presenta de acuerdo a la Figura 2.

En este caso, a diferencia de la vulnerabilidad física, más de un $90 \%$ de las manzanas estudiadas arrojó una vulnerabilidad baja. Lo anterior puede deberse a los resultados obtenidos en las variables de cantidad de población y viviendas por manzanas, como se explicó anteriormente.

Desde la perspectiva del Riesgo, los estudios realizados por el SHOA focalizan su atención en el factor amenaza (tsunami), orientándose específicamente a la determinación de los niveles máximos de inundación esperados para las principales zonas urbanas del borde costero de Chile, ante la ocurrencia de un tsunami o maremoto (ONEMI, 2001). De acuerdo a esto, se determinó la altitud mediante SIG utilizando un DEM Aster, donde se estableció la cota $30 \mathrm{msnm}$ y se seleccionaron las manzanas que se encontraban bajo esta altitud. Esto permitió trabajar sobre aquellas unidades que presentan algún tipo de riesgo. De acuerdo a lo descrito por Araya y Vergara (1976), la ciudad de San Antonio se configura en tres terrazas originadas por la abrasión marina: $200-250 \mathrm{msnm}$, para la terraza alta; $100-150 \mathrm{msnm}$, para la terraza media y $0-50 \mathrm{msnm}$, para la terraza baja.

Al relacionar las vulnerabilidades (física y socioeconómica y demográfica) con la amenaza, obtenemos el riesgo. La zonificación final permite observar un Riesgo de carácter alto para un 19\% del total de las manzanas. Este porcentaje hace referencia a una cantidad estimativa de 63 manzanas en un alto riesgo de inundación por tsunami. Con un porcentaje mayor se encuentra el riesgo considerado como medio, el cual presenta un $42 \%$ del total analizado, estimando una cantidad aproximada de 139 manzanas. Y por último, las manzanas consideradas en un riesgo bajo representan un 39\%, abarcando junto a ello un total de 130 manzanas (véase Figuras 3 y 4$)$.

\section{Conclusiones y recomendaciones}

La aplicación de esta metodología permitió identificar las zonas de riesgo de tsunami en la ciudad de San Antonio, donde de acuerdo a los resultados obtenidos, se pudo observar que sólo un 19\% de las manzanas analizadas presenta un riesgo alto, resultado influenciado en gran parte por la distancia de las viviendas al borde costero. En este caso, el puerto establece una distancia importante al inicio de la zona urbana. Sin embargo, se configura como otra variable a considerar cuando se evalúe 
el riesgo, ya que las estructuras utilizadas como conteiner y grúas aumentan el poder destructivo de un tsunami, una vez que son arrastradas por el mismo.

Ahora bien, por las características propias de su geografía, Chile es un país propenso al riesgo de tsunami. Desde 1570 han ocurrido 70 terremotos tsunamigénicos que han devastado las costas y su población. La necesidad de contar con información que ayude a la toma de decisiones en las primeras horas de ocurrida una emergencia se vuelve vital para el uso eficiente, eficaz y oportuno de los escasos recursos con los que cuentan los gobiernos locales como los municipios.

Es por esto, que la identificación de zonas con riesgo de desastres frente a determinados fenómenos naturales, ha sido un gran desafío para los organismos de respuestas, quienes buscan contar con información confiable que les permita tomar decisiones oportunas frente a situaciones de riesgo. Actualmente, en el país no existe una metodología estándar para la realización de mapas de riesgo, por lo que la diversificación y complejidad de metodologías ha dificultado su realización.

Las acciones de la primera respuesta frente a una emergencia, siempre estarán destinadas al rescate de vidas humanas, por lo que se vuelve primordial tener identificados aquellos lugares que necesitarán ayuda inmediata una vez ocurrido un desastre. Los tsunamis pueden ser muy devastadores, pero si se tienen las medidas necesarias de evacuación se puede evitar un aumento en el número de muertes que ha generado un terremoto.

El aporte de este trabajo se funda en la colaboración que representa para los gobiernos locales (municipios) contar con herramientas de fácil manejo que les permitan obtener información relevante para la toma de decisiones y planificación. Siendo esta jerarquía administrativa la primera en responder frente a situaciones emergencia, es común que los municipios no cuenten con instrumentos de zonificación de riesgos frente a determinados eventos. Si bien ha habido un avance importante en el desarrollo de información científica que ha permitido conocer mejor las amenazas de nuestro país, faltan instrumentos o herramientas que permitan relacionar estos estudios con variables de vulnerabilidad. La metodología propuesta presenta un desarrollo rápido, sencillo y con resultados medibles, considerando tan sólo seis variables de vulnerabilidad y una de amenaza, lo que permitió determinar cuántas manzanas están en un alto riesgo para la primera respuesta. Ahora bien, la incorporación de nuevas variables a la propuesta metodológica permitirá detallar con más precisión aquellos lugares que tienen un riesgo alto. Por ejemplo, la importancia del detalle de la población en referencia a los menores de 10 años y mayores de 65 años (población más vulnerable ante un tsunami) ayudaría en el enfoque principal en la primera respuesta.

Otra variable importante a considerar en la utilización de esta propuesta metodológica, es el cruce de los resultados con la capa de información que contenga el emplazamiento de los servicios públicos que participan en la gestión de la emergen- 
cia (carabineros, bomberos, centros de salud, albergues, entre otros). El cruce de estas variables contribuiría a la planificación de un plan de emergencia.

En el caso de la amenaza, dado que no existen cartas de inundación para todas las localidades costeras del país, en esta propuesta no se consideró su utilización. Sin embargo, en caso de existir, se recomienda utilizarla como referente para determinar la zona de inundación y acotar mejor la zona de amenaza.

\section{Bibliografía}

Araya-Vergara, J.F. (1976). "Reconocimiento de tipos e individuos geomorfológicos regionales en la costa de Chile", Revista Informaciones Geográficas, vol. 23, Chile, pp. 9-30.

Aster GDEM, NASA \& METI (octubre de 2011). DEM ASTER, Pasadena, California.

Biblioteca Congreso Nacional de Chile (2012). Disponible en $<\mathrm{http}: / /$ reportescomunales.bcn.cl/>, $<$ http://reportescomunales.bcn.cl/2012/index.php/San_Antonio/Poblaci\%C3\%B $3 \mathrm{n}>$

Cardona, O.D. (1991). "Evaluación de la Amenaza, la Vulnerabilidad y el Riesgo", Bogotá, Colombia.

Cardona, et al. (2013), "Modelación probabilista para la gestión del riesgo de desastre", Bogotá, Colombia.

Carvajal, L. (15 de diciembre de 2010). Disponible en <http://www. subdere.gov.cl>, obtenido de <http://www.subdere.gov.cl/sites/default/ noticiasold/articles-81926_recurso_14.ppsx $>$

Chardon y González (2002). Indicadores para la gestión de riesgos, Manizales, Colombia, IDEA UN.

CENEPRED (2013). Manual para la evaluación de riesgos originados por fenómenos naturales, Lima, Perú.

Centro de Información de Recursos Naturales —CIREN (2009). DEM ASTER CHILE, Santiago, Región Metropolitana, Chile.

Cortijo, O.L. (2009). Metodología para análisis de vulnerabilidad y riesgo ante inundaciones y sismos, de las edificaciones en centros urbanos, Lima.

Gómez, J.J. (2001). "Vulnerabilidad y medio ambiente", CEPAL, documento preparado para el Seminario internacional "Las diferentes expresiones de la vulnerabilidad social en América Latina y el Caribe", Santiago de Chile, 20 y 21 de junio.

Henoch, P. (2010). "Vulnerabilidad social. Más allá de la pobreza", Serie Informe Social, Libertad y Desarrollo, Santiago, Chile.

INDECI \& ITDG (2009). Gestión del riesgo de desastres, para la planificación del desarrollo local, Lima. 
INDECI (2006). Manual básico para la estimación del riesgo, Lima, Perú.

Istituto Nazionale Geofisica e Vulcanologia, I. (s.f.), INGV. Consultado en $<$ http://www.ingv.it/it/>

Lockridge, P. (1985). "Tsunamis in Chile-Peru”, Boulder: Report SE-39, Worl Data Centera for solid earth and geophysics.

Mardones, M. y Vidal, C. (2001). Zonificación y evaluación de los riesgos naturales de tipo geomorfológico: un instrumento para la planificación urbana en la ciudad de Concepción, Santiago, Chile.

Martínez, C. (2012). "Riesgo de tsunami en caleta Tubul, Región del Biobío: escenarios extremos y transformaciones territoriales posterremoto", Revista de Geografía Norte Grande, vol. 53, Santiago, Chile, pp. 85-106.

Monge, J. (1993). "Estudios de riesgo de tsunami en costas chilenas", Jornadas Chilenas de Sismología e Ingeniería Antisísmica, vol. 2, Santiago, Chile, pp. 3-22.

Ojeda, G., lacreu, H., y Sosa, G. (2007). Atlas de Recursos Geoambientales. Potrero de los Funes, Universidad Nacional de San Luis, San Luis, Argentina.

ONEMI (2001). Metodología básica para la elaboración de un plan común de prevención y de respuesta ante un Tsunami, Santiago, Chile.

(12 de marzo de 2002). Disponible en <http://www.intendenciamaule. gov.cl>, <http://www.intendenciamaule.gov.cl>

(2014). Política Nacional para la Gestión del Riesgo de Desastres”, Santiago, Chile.

Parga, V. (2010). Evaluación de la construcción social del riesgo frente a amenazas de tsunami en el área urbana de la comuna de caldera, III Región de Atacama, Santiago, Chile.

Paton, D. and Johnston, D. (2006). Disaster Resilience, An Integrated Approach, Illinois, Charles C. Thomas Publisher Ltda.

PRC San Antonio (2006). Consultado en <www.sanantonio.cl>, recuperado el 04 de junio de 2014.

Sen, A. (1988). The Concept of Development, Amsterdam, North Holland, H. Chenery and T.N. Srinivasan.

Servicio Hidrográfico y Oceanográfico de la Armada - SHOA (23 de febrero de 2004). Recuperado el 01 de junio de 2014, <www.shoa.cl $>$ (2012). Tsunami, Las grandes olas, Santiago, Chile.

SiTsA (2014). Coberturas San Antonio, San Antonio, Región de Valparaíso, Chile. Strahler, A. (1994). Geografía Física, Editorial Omega, Barcelona, España.

Tarbuck, E., and Lutgens, F. (2005). Ciencias de la tierra: una introducción a la geología física, Pearson Educación, Madrid, España.

UNIDSR (2010). Diagnóstico de la situación de la reducción del riesgo de desastres en Chile, Oficina Regional de Naciones Unidas, Panamá. 
Vargas, J. (2002). Políticas públicas para la reducción de la vulnerabilidad frente a los desastres naturales y socio naturales, División de Medio Ambiente y Asentamientos Humanos, Santiago, Chile.

Wiegel, R. (1970). Earthquake engineering, Englewood Cliffs, Prentice-Hall.

Wilches-chaux, G. (1984). Tipos de Vulnerabilidad, Servicio Nacional de Aprendizaje, Popayán. 